\title{
Internal and Emergency Medicine (IEM) has obtained its fourth impact factor
}

\author{
Domenico Prisco
}

Published online: 2 July 2013

(c) SIMI 2013

Dear readers,

Internal and Emergency Medicine (IEM) has obtained its fourth impact factor (IF), with a respectable 2.345, which is a significant improvement over last year's IF of 2.057. During 2012, IEM gained 347 citations for the 148 articles published in 2010 and 2011 vs. the 216 citations obtained in 2011 for the 105 articles published in 2009 and 2010. I emphasize that the corresponding figures for 2010 were: 154 citations for 72 articles published in 2008 and 2009. In practice the articles considered by ISI were 28 in 2007, 34 in 2008, 38 in 2009, 67 in 2010 and 81 in 2011 with a continuous and very marked increase in the number of papers reviewed and articles published! Nevertheless, despite such an increase in published articles, it is clear that the number of citations needs time to increase. Accordingly, with the improvement in the IF, we anticipate even further citations in the future.

Our Journal is ranked 33th out of 151 journals in the category Medicine General and Internal (last year it was 40th out of 153). Over the past year, we have continued to receive increasing numbers of papers from many countries.
Most importantly, downloads of our articles have progressively increased to demonstrate the relevance of our papers for scientific community.

The mean number of submissions per year appears to have stabilized at about 450 . The mean rejection rate is still 70-80\%, and we will publish review articles only when they have been solicited by invitation.

We have significantly reduced the number of Letters and Medical Illustrations, and we schedule case records upon invitation according to proposals we receive from our readers.

Lastly, a warm special thanks to Peter Rosen and the group of the Emergency Medicine section for their very appreciated work for the Journal. I thank also the members of different boards, the president and the council of SIMI and the publisher Springer.

With the end of 2013, I am at the end of my term. The first years with Gian Franco Gensini and the past ones as editor-in-chief have been very rich positive experiences, and I thank you, our readers, for your support.

Domenico Prisco

Editor-in-Chief

Internal and Emergency Medicine

\footnotetext{
D. Prisco ( $\square)$

Internal and Emergency Medicine, Florence, Italy

e-mail: priscod@aou-careggi.toscana.it

D. Prisco

Department of Experimental and Clinical Medicine,

University of Florence, Florence, Italy

D. Prisco

Patologia Medica Unit, Careggi University Hospital,

Florence, Italy
} 